\title{
On common fixed point theorems for $(\psi, \phi)$-generalized $f$-weakly contractive mappings
}

\author{
H. Aydi
}




\title{
ON COMMON FIXED POINT THEOREMS FOR $(\psi, \varphi)$-GENERALIZED $f$-WEAKLY CONTRACTIVE MAPPINGS
}

\author{
H. AYDI
}

Received September 15, 2011

\begin{abstract}
In this paper, we present some common fixed point theorems for $(\psi, \varphi)$-generalized $f$ weakly contractive mappings in metric and ordered metric spaces. Our results extend, generalize and improve some well-known results in the literature. Also, we give an example to illustrate our results.
\end{abstract}

2000 Mathematics Subject Classification: 54H25; 47H10; 54E50

Keywords: common fixed point, commuting maps, $f$-weakly contractive maps, generalized $f$ weakly contractive maps, ordered metric space

\section{INTRODUCTION AND PRELIMINARIES}

The first important result on fixed points for contractive type mapping was the much celebrated Banach's contraction principle by Banach [2] in 1922. After this, Kannan $[9,10]$ proved the following result:

Theorem 1. Let $(X, d)$ be a complete metric space. If $T: X \rightarrow X$ satisfies

$$
d(T x, T y) \leq k[d(x, T x)+d(y, T y)]
$$

where $0<k<\frac{1}{2}$ and $x, y \in X$, then $T$ has a unique fixed point.

A similar type of contractive condition has been studied by Chatterjee [5] and he proved the following result:

Theorem 2. Let $(X, d)$ be a complete metric space. If $T: X \rightarrow X$ satisfies a $C$-contraction given as follows:

$$
d(T x, T y) \leq k[d(x, T y)+d(y, T x)],
$$

where $0<k<\frac{1}{2}$ and $x, y \in X$, then $T$ has a unique fixed point.

Alber and Guerre-Delabriere [1] introduced the definition of weak $\Phi$-contraction.

Definition 1. A self mapping $T$ on a metric space $X$ is called weak $\Phi$-contraction if there exists a function $\Phi:[0,+\infty) \rightarrow[0,+\infty)$ such that for each $x, y \in X$,

$$
d(T x, T y) \leq d(x, y)-\Phi(d(x, y))) .
$$

(C) 2013 Miskolc University Press 
The notion of $\Phi$-contraction and weak $\Phi$-contraction has been studied by many authors, see $[3,12,15,17,19]$. In recent years, many results related to fixed point theorems in partially ordered metric spaces are given, for more details see [8,12-16].

Choudhury in [6] introduced a generalization of $C$-contraction given by the following definition.

Definition 2 ([6]). Let $(X, d)$ be a metric space. A mapping $T: X \rightarrow X$ is said to be weakly $C$-contractive (or a weak $C$-contraction) if for all $x, y \in X$,

$$
d(T x, T y) \leq \frac{1}{2}(d(x, T y)+d(y, T x))-\varphi(d(x, T y), d(y, T x)),
$$

where $\varphi:[0,+\infty) \rightarrow[0,+\infty)$ is a continuous function such that $\varphi(x, y)=0$ if and only $x=y=0$.

In [6] the author proves that if $X$ is complete then every weak $C$-contraction has a unique fixed point. Recently, Harjani et al, [8] presented this last result in the context of ordered metric spaces.

Chandok [4] introduced the following definition: A map $T: X \rightarrow X$ is generalized $f$-weakly contractive if for each $x, y \in X$,

$$
d(T x, T y) \leq \frac{1}{2}(d(f x, T y)+d(f y, T x))-\varphi(d(f x, T y), d(f y, T x)),
$$

where $\varphi:[0,+\infty) \rightarrow[0,+\infty) \rightarrow[0,+\infty)$ is a continuous function such that $\varphi(x, y)=$ 0 if and only $x=y=0$.

If $f=I_{X}$, the identity mapping, then generalized $f$-weakly contractive mapping is weakly $C$-contractive.

Khan et al. [11] introduced the concept of altering distance function as follows:

Definition 3 (altering distance function, [11]). The function $\psi:[0,+\infty) \rightarrow[0,+\infty)$ is called an altering distance function if the following properties are satisfied:

(1) $\psi$ is continuous and non-decreasing.

(2) $\psi(t)=0$ if and only if $t=0$.

Following the above definitions, we introduce the following:

Definition 4. A map $T: X \rightarrow X$ is called $(\psi, \varphi)$-generalized $f$-weakly contractive if for each $x, y \in X$,

$$
\psi(d(T x, T y)) \leq \psi\left(\frac{1}{2}[d(f x, T y)+d(f y, T x)]\right)-\varphi(d(f x, T y), d(f y, T x)),
$$

where

(1) $\psi:[0,+\infty) \rightarrow[0,+\infty)$ is an altering distance function.

(2) $\varphi:[0,+\infty) \times[0,+\infty) \rightarrow[0,+\infty)$ is a continuous function with $\varphi(t, s)=0$ if and only if $t=s=0$. 
If $\psi(t)=t$, then $(\psi, \varphi)$-generalized $f$-weakly contractive mapping is generalized $f$-weakly contractive.

The aim of this paper is to study some common fixed point theorems for $(\psi, \varphi)$ generalized $f$-weakly contractive in metric and ordered metric spaces.

\section{MAIN RESUlTS}

First, we state the following known definition:

Definition 5. Let $X$ a non-empty set. A point $x \in X$ is a coincidence point (common fixed point) of $f: X \rightarrow X$ and $T: X \rightarrow X$ if $f x=T x(x=f x=T x)$. The pair $\{f, T\}$ is called commuting if $T f x=f T x$ for all $x \in X$.

We start with a common fixed point theorem for $(\psi, \varphi)$-generalized $f$-weakly contractive mappings in complete metric spaces.

Theorem 3. Let $(X, d)$ be a metric space. Let $f, T: X \rightarrow X$ satisfy $T(X) \subset f(X)$, $(f(X), d)$ is complete and

$$
\psi(d(T x, T y)) \leq \psi\left(\frac{1}{2}[d(f x, T y)+d(f y, T x)]\right)-\varphi(d(f x, T y), d(f y, T x)),
$$

for all $x, y \in X$, where

(1) $\psi:[0,+\infty) \rightarrow[0,+\infty)$ is an altering distance function,

(2) $\varphi:[0,+\infty) \times[0,+\infty) \rightarrow[0,+\infty)$ is a continuous function with $\varphi(t, s)=0$ if and only if $t=s=0$, then $T$ and $f$ have a coincidence point in $X$. Further, if $T$ and $f$ commute at their coincidence points, then $T$ and $f$ have a common fixed point.

Proof. Let $x_{0} \in X$. Since $T(X) \subset f(X)$, we can choose $x_{1} \in X$, so that $f x_{1}=$ $T x_{0}$. Since $T x_{1} \in f(X)$, there exists $x_{2} \in X$ such that $f x_{2}=T x_{1}$. By induction, we construct a sequence $\left\{x_{n}\right\}$ in $X$ such that $f x_{n+1}=T x_{n}$, for every $n \in \mathbb{N}$. By inequality (2.1), we have

$$
\begin{aligned}
\psi\left(d\left(T x_{n+1}, T x_{n}\right)\right) \leq & \psi\left(\frac{1}{2}\left[d\left(f x_{n+1}, T x_{n}\right)+d\left(f x_{n}, T x_{n+1}\right)\right]\right) \\
& -\varphi\left(d\left(f x_{n+1}, T x_{n}\right), d\left(f x_{n}, T x_{n+1}\right)\right) \\
= & \psi\left(\frac{1}{2} d\left(T x_{n-1}, T x_{n+1}\right)\right)-\varphi\left(0, d\left(T x_{n-1}, T x_{n+1}\right)\right) \\
\leq & \psi \\
\leq & \left.\frac{1}{2} d\left(T x_{n-1}, T x_{n+1}\right)\right) \\
\leq & =\psi\left(\frac{1}{2}\left[d\left(T x_{n-1}, T x_{n}\right)+d\left(T x_{n}, T x_{n+1}\right)\right]\right)
\end{aligned}
$$

Since $\psi$ is a non-decreasing function, we get that

$$
d\left(T x_{n}, T x_{n+1}\right) \leq d\left(T x_{n-1}, T x_{n}\right) \quad \text { for any } n \in \mathbb{N}^{*} .
$$


Thus, $\left\{d\left(T x_{n}, T x_{n+1}\right)\right\}$ is a monotone non-increasing sequence of non-negative real numbers and hence is convergent. Hence there is $r \geq 0$ such that

$$
\lim _{n \rightarrow+\infty} d\left(T x_{n}, T x_{n+1}\right)=r .
$$

Using a triangular inequality, we have

$$
d\left(T x_{n+1}, T x_{n}\right) \leq \frac{1}{2} d\left(T x_{n-1}, T x_{n+1}\right) \leq \frac{1}{2}\left[d\left(T x_{n-1}, T x_{n}\right)+d\left(T x_{n}, T x_{n+1}\right)\right] .
$$

Letting $n \rightarrow+\infty$, we get

$$
r \leq \frac{1}{2} \lim _{n \rightarrow+\infty} d\left(T x_{n-1}, T x_{n+1}\right) \leq \frac{1}{2} r+\frac{1}{2} r,
$$

that is $\lim _{n \rightarrow+\infty} d\left(T x_{n-1}, T x_{n+1}\right)=2 r$. Using the continuity of $\psi$ and $\varphi$, and inequality (2.2), we have, letting $n \rightarrow+\infty$

$$
\psi(r) \leq \psi(r)-\varphi(0,2 r),
$$

and consequently, $\varphi(0,2 r) \leq 0$. Thus, by a property of $\varphi, r=0$, so

$$
\lim _{n \rightarrow+\infty} d\left(T x_{n+1}, T x_{n}\right)=0 .
$$

Now, we show that $\left\{T x_{n}\right\}$ is a Cauchy sequence. If otherwise, then there exists $\varepsilon>0$ for which we can find subsequences $\left\{T x_{m(k)}\right\}$ and $\left\{T x_{n(k)}\right\}$ of $\left\{T x_{n}\right\}$ with $n(k)>m(k)>k$ such that for every $k$,

$$
d\left(T x_{m(k)}, T x_{n(k)}\right) \geq \varepsilon, \quad d\left(T x_{m(k)}, T x_{n(k)-1}\right)<\varepsilon .
$$

By triangular inequality, we have from (2.5)

$$
\begin{aligned}
\varepsilon & \leq d\left(T x_{m(k)}, T x_{n(k)}\right) \\
& \leq d\left(T x_{m(k)}, T x_{n(k)-1}\right)+d\left(T x_{n(k)-1}, T x_{n(k)}\right) \\
& <\varepsilon+d\left(T x_{n(k)-1}, T x_{n(k)}\right) .
\end{aligned}
$$

Using (2.4), we get

$$
\lim _{k \rightarrow+\infty} d\left(T x_{m(k)}, T x_{n(k)}\right)=\lim _{k \rightarrow+\infty} d\left(T x_{m(k)}, T x_{n(k)-1}\right)=\varepsilon .
$$

On the other hand,

$$
\begin{aligned}
d\left(T x_{m(k)}, T x_{n(k)-1}\right) \leq & d\left(T x_{m(k)}, T x_{m(k)-1}\right)+d\left(T x_{m(k)-1}, T x_{n(k)}\right) \\
& +d\left(T x_{n(k)}, T x_{n(k)-1}\right)
\end{aligned}
$$

and

$$
d\left(T x_{m(k)-1}, T x_{n(k)}\right) \leq d\left(T x_{m(k)-1}, T x_{m(k)}\right)+d\left(T x_{m(k)}, T x_{n(k)}\right) .
$$

Letting $k \rightarrow+\infty$ in the two above inequalities, we have thanks to (2.4) and (2.6),

$$
\lim _{k \rightarrow+\infty} d\left(T x_{m(k)-1}, T x_{n(k)}\right)=\varepsilon .
$$


From (2.1), we have

$$
\begin{aligned}
\psi(\varepsilon) \leq & \psi\left(d\left(T x_{m(k)}, T x_{n(k)}\right)\right) \\
\leq & \psi\left(\frac{1}{2}\left[d\left(f x_{m(k)}, T x_{n(k)}\right)+d\left(f x_{n(k)}, T x_{m(k)}\right)\right]\right) \\
& -\varphi\left(d\left(f x_{m(k)}, T x_{n(k)}\right), d\left(f x_{n(k)}, T x_{m(k)}\right)\right) \\
= & \psi\left(\frac{1}{2}\left[d\left(T x_{m(k)-1}, T x_{n(k)}\right)+d\left(T x_{n(k)-1}, T x_{m(k)}\right)\right]\right) \\
& -\varphi\left(d\left(T x_{m(k)-1}, T x_{n(k)}\right), d\left(T x_{n(k)-1}, T x_{m(k)}\right)\right) .
\end{aligned}
$$

Taking $k \rightarrow+\infty$, using the continuity of $\psi$ and $\varphi$, we obtain from (2.6), (2.7)

$$
\psi(\varepsilon) \leq \psi(\varepsilon)-\varphi(\varepsilon, \varepsilon),
$$

hence $\varphi(\varepsilon, \varepsilon)=0$, so $\varepsilon=0$, it is a contradiction. Thus $\left\{T x_{n}\right\}$ is a Cauchy sequence. Since $f x_{n}=T x_{n-1}$, hence $\left\{f x_{n}\right\}$ is a Cauchy sequence in $(f(X), d)$, which is complete. Thus there is $z \in X$ such that

$$
\lim _{n \rightarrow+\infty} f x_{n}=f z
$$

Moreover, (2.4) reads

$$
\lim _{n \rightarrow+\infty} d\left(f x_{n}, f x_{n+1}\right)=0
$$

By (2.1), we have

$$
\begin{aligned}
& \psi\left(d\left(T z, f x_{n+1}\right)\right)=\psi\left(d\left(T z, T x_{n}\right)\right) \\
& \leq \psi\left(\frac{1}{2}\left[d\left(f z, T x_{n}\right)+d\left(f x_{n}, T z\right)\right]\right)-\varphi\left(d\left(f z, T x_{n}\right), d\left(f x_{n}, T z\right)\right) \\
& =\psi\left(\frac{1}{2}\left[d\left(f z, f x_{n+1}\right)+d\left(f x_{n}, T z\right)\right]\right)-\varphi\left(d\left(f z, f x_{n+1}\right), d\left(f x_{n}, T z\right)\right),
\end{aligned}
$$

and letting $n \rightarrow+\infty$, using the continuity of $\psi$ and $\varphi$ and by (2.8), (2.9), we find

$$
\psi(d(T z, f z)) \leq \psi\left(\frac{1}{2} d(T z, f z)\right)-\varphi(0, d(f z, T z)) \leq \psi\left(\frac{1}{2} d(T z, f z)\right) .
$$

Consequently, $d(T z, f z) \leq \frac{1}{2} d(T z, f z)$, that is, $d(T z, f z)=0$, i,e. $T z=f z$, hence $z$ is a coincidence point of $T$ and $f$. Now suppose that $T$ and $f$ commute at $z$. Let $w=T z=f z$. Then $T w=T(f z)=f(T z)=f w$. By inequality (2.1)

$$
\begin{aligned}
\psi(d(T z, T w) & \leq \psi\left(\frac{1}{2}[d(f z, T w)+d(f w, T z)]\right)-\varphi(d(f z, T w), d(f w, T z)) \\
& =\psi\left(\frac{1}{2}[d(T z, T w)+d(T w, T z)]\right)-\varphi(d(T z, T w), d(T w, T z)) \\
& =\psi\left(\frac{1}{2}[d(T z, T w)+d(T w, T z)]\right)-\varphi(d(T z, T w), d(T w, T z)) \\
& =\psi(d(T z, T w))-\varphi(d(T z, T w), d(T w, T z)) .
\end{aligned}
$$


This implies that $d(T z, T w)=0$, by the property of $\varphi$. Therefore, $T w=f w=w$. This completes the proof of Theorem 3 .

Example 1. Let $X=[0,+\infty)$. Let $d$ be defined by $d(x, y)=|x-y|$ for all $x, y \in X$. We set $f x=\frac{x}{2}$ and $T x=\frac{x}{4}$ for all $x \in X$. It is clear that $T(X) \subset f(X)$ and $(f(X), d)$ is a complete metric space. Define $\psi:[0,+\infty) \rightarrow[0,+\infty)$ and $\varphi$ : $[0,+\infty) \times[0,+\infty) \rightarrow[0,+\infty)$ by

$$
\psi(t)=\frac{t}{2} \quad \text { and } \quad \varphi(t, s)=\frac{1}{16}(t+s) .
$$

It is obvious that $\psi$ and $\varphi$ satisfy the hypotheses of Theorem 3 . We need to show that the inequality (2.1) holds for any $x, y \in X$. First, the left-hand side of (2.1) is

$$
\psi(d(T x, T y))=\frac{1}{8}|x-y| .
$$

While, the right-hand side of (2.1) is

$$
\begin{aligned}
& \psi\left(\frac{1}{2}(d(f x, T y)+d(f y, T x))-\varphi(d(f x, T y), d(f y, T x)\right. \\
& =\frac{1}{4}\left[\left|\frac{x}{2}-\frac{y}{4}\right|+\left|\frac{y}{2}-\frac{x}{4}\right|\right]-\frac{1}{16}\left[\left|\frac{x}{2}-\frac{y}{4}\right|+\left|\frac{y}{2}-\frac{x}{4}\right|\right] \\
& =\frac{3}{16}\left[\left|\frac{x}{2}-\frac{y}{4}\right|+\left|\frac{y}{2}-\frac{x}{4}\right|\right] .
\end{aligned}
$$

By symmetry of (2.10) and (2.11), and without loss of generality, we suppose that $x \geq y$. In particular, (2.10) reads

$$
\psi(d(T x, T y))=\frac{1}{8}(x-y) .
$$

We distinguish two cases:

- If $2 y \geq x$. Here, we have from (2.11)

$$
\begin{aligned}
& \psi\left(\frac{1}{2}(d(f x, T y)+d(f y, T x))-\varphi(d(f x, T y), d(f y, T x)\right. \\
& =\frac{3}{16}\left[\left|\frac{x}{2}-\frac{y}{4}\right|+\left|\frac{y}{2}-\frac{x}{4}\right|\right]=\frac{3}{16}\left[\left(\frac{x}{2}-\frac{y}{4}\right)+\left(\frac{y}{2}-\frac{x}{4}\right)\right] \\
& =\frac{3}{64}(x+y) \geq \frac{1}{8}(x-y)=\psi(d(T x, T y)) .
\end{aligned}
$$

- If $2 y<x$. Here, we have from (2.11)

$$
\begin{aligned}
& \psi\left(\frac{1}{2}(d(f x, T y)+d(f y, T x))-\varphi(d(f x, T y), d(f y, T x)\right. \\
& =\frac{3}{16}\left[\left|\frac{x}{2}-\frac{y}{4}\right|+\left|\frac{y}{2}-\frac{x}{4}\right|\right]=\frac{3}{16}\left[\left(\frac{x}{2}-\frac{y}{4}\right)+\left(-\frac{y}{2}+\frac{x}{4}\right)\right] \\
& =\frac{9}{64}(x-y) \geq \frac{1}{8}(x-y)=\psi(d(T x, T y)) .
\end{aligned}
$$


By (2.12) and (2.13), the inequality (2.1) is satisfied. Then by Theorem 3, $T$ and $f$ have a common fixed point, which is $z=0$.

Corollary 1. Let $(X, d)$ be a complete metric space. If $T: X \rightarrow X$ satisfies

$$
\psi(d(T x, T y)) \leq \psi\left(\frac{1}{2}[d(x, T y)+d(y, T x)]\right)-\varphi(d(x, T y), d(y, T x)),
$$

for all $x, y \in X$, where

(1) $\psi:[0,+\infty) \rightarrow[0,+\infty)$ is an altering distance function,

(2) $\varphi:[0,+\infty) \times[0,+\infty) \rightarrow[0,+\infty)$ is a continuous function with $\varphi(t, s)=0$ if and only if $t=s=0$, then $T$ has a unique fixed point.

Proof. It follows by taking $f=I_{X}$ in Theorem 3. The uniqueness of the fixed point follows by the following: suppose $u$ and $v$ are fixed points of $T$. By (2.14), we have

$$
\begin{aligned}
\psi(d(u, v)) & =\psi(d(T u, T v)) \\
& \leq \psi\left(\frac{1}{2}[d(u, T v)+d(v, T u)]\right)-\varphi(d(u, T v), d(v, T u)) \\
& =\psi\left(\frac{1}{2}[d(u, v)+d(v, u)]\right)-\varphi(d(u, v), d(v, u)) \\
& =\psi(d(u, v))-\varphi(d(u, v), d(v, u)),
\end{aligned}
$$

which implies that $\varphi(d(u, v), d(v, u))=0$, and by a property of $\varphi$, we get $u=v$.

Corollary 2. Let $(X, d)$ be a metric space. If $T, f: X \rightarrow X$ are such that $T(X) \subset$ $f(X),(f(X), d)$ is complete and

$$
d(T x, T y) \leq \frac{1}{2}[d(f x, T y)+d(f y, T x)]-\varphi(d(f x, T y), d(f y, T x)),
$$

for all $x, y \in X$, where $\varphi:[0,+\infty) \times[0,+\infty) \rightarrow[0,+\infty)$ is a continuous function with $\varphi(t, s)=0$ if and only if $t=s=0$, then $T$ and $f$ have a coincidence point in $X$. Further, if $T$ and $f$ commute at their coincidence points, then $T$ and $f$ have $a$ common fixed point.

Proof. The proof follows by taking $\psi(t)=t$ in Theorem 3 .

Corollary 3. Let $(X, d)$ be a complete metric space. If $T: X \rightarrow X$ satisfies for all $x, y \in X$

$$
d(T x, T y) \leq \frac{1}{2}[d(x, T y)+d(y, T x)]-\varphi(d(x, T y), d(y, T x)),
$$

where $\varphi:[0,+\infty) \times[0,+\infty) \rightarrow[0,+\infty)$ is a continuous function with $\varphi(t, s)=0$ if and only if $t=s=0$, then $T$ has a unique fixed point.

Proof. It follows by taking $f=I d_{X}$ in Corollary 2. The uniqueness of the fixed point follows from Corollary 1. 
Remark 1. - Corollary 1 corresponds to Corollary 2.1 of Shatanawi [18].

- Corollary 2 corresponds to Theorem 1 of Chandok [4].

- Corollary 3 corresponds to Theorem 2.1 of Choudhury [6].

Now, we extend Theorem 3 and we prove a common fixed point theorem for $f$ non-decreasing generalized nonlinear contraction mappings in the context of ordered metric spaces.

Definition 6 ([7]). Suppose $(X, \leq)$ is a partially ordered set and $T, f: X \rightarrow X . T$ is said to be monotone $f$-nondecreasing if for all $x, y \in X$,

$$
f x \leq f y \text { implies } T x \leq T y .
$$

If $f=I_{X}$ in Definition 6, then $T$ is monotone non-decreasing.

Theorem 4. Let $(X, \leq)$ be a partially ordered set and suppose that there exists a metric $d$ on $X$ such that $(X, d)$ is a complete metric space. Let $f$ and $T$ are selfmappings of $X$ such that $T(X) \subset f(X), f(X)$ is closed and $T$ is $f$-non-decreasing mapping. Suppose that $f$ and $T$ satisfy for all $x, y \in X$, for which $f(x) \leq f(y)$

$$
\psi(d(T x, T y)) \leq \psi\left(\frac{1}{2}[d(f x, T y)+d(f y, T x)]\right)-\varphi(d(f x, T y), d(f y, T x))
$$

where

(1) $\psi:[0,+\infty) \rightarrow[0,+\infty)$ is an altering distance function,

(2) $\varphi:[0,+\infty) \times[0,+\infty) \rightarrow[0,+\infty)$ is a continuous function with $\varphi(x, y)=0$ if and only if $x=y=0$.

Also, suppose that if $\left\{f\left(x_{n}\right)\right\} \subset X$ is a non-decreasing sequence with $f\left(x_{n}\right) \rightarrow f(z)$ in $f(X)$, then $f\left(x_{n}\right) \leq f(z)$ and $f(z) \leq f(f(z))$ for every $n$.

If there exists $x_{0} \in X$ with $f x_{0} \leq T x_{0}$, then $T$ and $f$ have a coincidence point. Further, if $T$ and $f$ commute at their coincidence points, then $T$ and $f$ have $a$ common fixed point.

Proof. Let $x_{0} \in X$ such that $f x_{0} \leq T x_{0}$. Since $T(X) \subset f(X)$, we can choose $x_{1} \in X$, so that $f x_{1}=T x_{0}$. Since $T x_{1} \in f(X)$, there exists $x_{2} \in X$ such that $f x_{2}=T x_{1}$. By induction, we construct a sequence $\left\{x_{n}\right\}$ in $X$ such that

$$
f x_{n+1}=T x_{n} .
$$

Since $f x_{0} \leq T x_{0}, T x_{0}=f x_{1}$, so $f x_{0} \leq f x_{1} . T$ is $f$-non-decreasing mapping, we get $T x_{0} \leq T x_{1}$. Similarly $f x_{1} \leq f x_{2}, T x_{1} \leq T x_{2}$, hence $f x_{2} \leq f x_{3}$. Continuing, we obtain

$$
f x_{0} \leq f x_{1} \leq f x_{2} \leq \ldots \leq f x_{n} \leq f x_{n+1} \leq \ldots
$$

If for some $n, T x_{n+1}=T x_{n}$, then $T x_{n+1}=f x_{n+1}$, i.e. $T$ and $f$ have a coincidence point $x_{n+1}$, and so we have the result. For the rest, assume that $d\left(T x_{n}, T x_{n+1}\right)>0$ 
for all $n \in \mathbb{N}$. By (2.18), we have

$$
\begin{aligned}
\psi\left(d\left(T x_{n}, T x_{n+1}\right)\right) \leq & \psi\left(\frac{1}{2}\left[d\left(f x_{n+1}, T x_{n}\right)+d\left(f x_{n}, T x_{n+1}\right)\right]\right) \\
& -\varphi\left(d\left(f x_{n+1}, T x_{n}\right), d\left(f x_{n}, T x_{n+1}\right)\right) \\
= & \psi\left(\frac{1}{2} d\left(T x_{n-1}, T x_{n+1}\right)\right)-\varphi\left(0, d\left(T x_{n-1}, T x_{n+1}\right)\right) \\
\leq & \psi\left(\frac{1}{2} d\left(T x_{n-1}, T x_{n+1}\right)\right) \\
\leq & \psi\left(\frac{1}{2} d\left(T x_{n-1}, T x_{n}\right)+\frac{1}{2} d\left(T x_{n}, T x_{n+1}\right)\right) .
\end{aligned}
$$

It follows that, for any $n \in \mathbb{N}^{*}$

$$
d\left(T x_{n}, T x_{n+1}\right) \leq d\left(T x_{n-1}, T x_{n}\right) .
$$

Thus $\left\{d\left(T x_{n}, T x_{n+1}\right)\right\}$ is a monotone non-increasing sequence, hence it is convergent. Now, proceeding as in Theorem 3, we can prove that

$$
\lim _{n \rightarrow+\infty} d\left(T x_{n}, T x_{n+1}\right)=0 \text {. }
$$

Moreover, $\left\{T x_{n}\right\}$ is a Cauchy sequence. Since $T x_{n}=f x_{n+1}$ and $f(X)$ is closed, so there exists $z \in X$ such that

$$
\lim _{n \rightarrow+\infty} f x_{n}=f z
$$

Having in mind $\left\{f x_{n}\right\}$ is a non-decreasing sequence, so by (2.20) we have for every $n \in \mathbb{N}$

$$
f x_{n} \leq f z, \quad f(z) \leq f(f z)
$$

Having $f x_{n} \leq f z$, so from inequality (2.18), we have

$$
\begin{aligned}
& \psi\left(d\left(f x_{n+1}, T z\right)\right)=\psi\left(d\left(T x_{n}, T z\right)\right) \\
& \leq \psi\left(\frac{1}{2}\left[d\left(f z, T x_{n}\right)+d\left(f x_{n}, T z\right)\right]\right)-\varphi\left(d\left(f z, T x_{n}\right), d\left(f x_{n}, T z\right)\right) \\
& =\psi\left(\frac{1}{2}\left[d\left(f z, f x_{n+1}\right)+d\left(f x_{n}, T z\right)\right]-\varphi\left(d\left(f z, f x_{n+1}\right), d\left(f x_{n}, T z\right)\right) .\right.
\end{aligned}
$$

Taking $n \rightarrow+\infty$, using the continuity of $\psi$ and $\varphi$, we get from (2.19), (2.20)

$$
\psi(d(T z, f z)) \leq \psi\left(\frac{1}{2} d(f z, f z)\right)-\varphi(0, d(f z, T z)),
$$

that is, $d(T z, f z)=0$, hence $T z=f z$, so $z$ is a coincidence point of $T$ and $f$.

Now suppose that $T$ and $f$ commute at $z$. Let $w=T z=f z$. Then $T w=$ $T(f z)=f(T z)=f w$. From (2.21), we have $f z \leq f(f z)=f w$, so the inequality (2.18) gives us

$$
\psi\left(d(T z, T w) \leq \psi\left(\frac{1}{2}[d(f z, T w)+d(f w, T z)]\right)-\varphi(d(f z, T w), d(f w, T z))\right.
$$




$$
\begin{aligned}
& =\psi\left(\frac{1}{2}[d(T z, T w)+d(T w, T z)]\right)-\varphi(d(T z, T w), d(T w, T z)) \\
& =\psi\left(\frac{1}{2}[d(T z, T w)+d(T w, T z)]\right)-\varphi(d(T z, T w), d(T w, T z)) \\
& =\psi(d(T z, T w))-\varphi(d(T z, T w), d(T w, T z)) .
\end{aligned}
$$

This implies that $d(T z, T w)=0$, by the property of $\varphi$. Therefore, $T w=f w=w$. This completes the proof of Theorem 4.

Corollary 4. Let $(X, \leq)$ be a partially ordered set and suppose that there exists a metric $d$ on $X$ such that $(X, d)$ is a complete metric space. Let $f$ and $T$ are selfmappings of $X$ such that $T(X) \subset f(X), f(X)$ is closed and $T$ is $f$-non-decreasing mapping. Assume that $f$ and $T$ satisfy for all $x, y \in X$, for which $f(x) \leq f(y)$

$$
d(T x, T y) \leq \frac{1}{2}[d(f x, T y)+d(f y, T x)]-\varphi(d(f x, T y), d(f y, T x)),
$$

where $\varphi:[0,+\infty) \times[0,+\infty) \rightarrow[0,+\infty)$ is a continuous function with $\varphi(x, y)=0$ if and only if $x=y=0$.

Also, suppose that if $\left\{f\left(x_{n}\right)\right\} \subset X$ is a non-decreasing sequence with $f\left(x_{n}\right) \rightarrow f(z)$ in $f(X)$, then $f\left(x_{n}\right) \leq f(z)$ and $f(z) \leq f(f(z))$ for every $n$.

If there exists $x_{0} \in X$ with $f x_{0} \leq T x_{0}$, then $T$ and $f$ have a coincidence point. Further, if $T$ and $f$ commute at their coincidence points, then $T$ and $f$ have a common fixed point.

Proof. It follows by taking $\psi(t)=t$ in Theorem 4 .

Corollary 5. Let $(X, \leq)$ be a partially ordered set and suppose that there exists a metric $d$ on $X$ such that $(X, d)$ is a complete metric space. Let $T: X \rightarrow X$ be a monotone non-decreasing mapping. Suppose that $T$ satisfies for all $x, y \in X$, for which $x \leq y$,

$$
\psi(d(T x, T y)) \leq \psi\left(\frac{1}{2}[d(x, T y)+d(y, T x)]\right)-\varphi(d(x, T y), d(y, T x)),
$$

where

(1) $\psi:[0,+\infty) \rightarrow[0,+\infty)$ is an altering distance function,

(2) $\varphi:[0,+\infty) \times[0,+\infty) \rightarrow[0,+\infty)$ is a continuous function with $\varphi(x, y)=0$ if and only if $x=y=0$.

Also suppose either

(i) $\left\{x_{n}\right\} \subset X$ is a non-decreasing sequence with $x_{n} \rightarrow z$, then $x_{n} \leq z$ for every $n$, or

(ii) $T$ is continuous.

If there exists $x_{0} \in X$ with $x_{0} \leq T x_{0}$, then $T$ has a fixed point. 
Proof. If (i) holds, then taking $f=I_{X}$ in Theorem 4, we get the result.

If (ii) holds, then proceeding as in Theorem 4 with $f=I_{X}$, we can prove that $\left\{T x_{n}\right\}$ is a Cauchy sequence and

$$
z=\lim _{n \rightarrow+\infty} x_{n+1}=\lim T x_{n}=T\left(\lim _{n \rightarrow+\infty} x_{n}\right)=T z .
$$

Hence the proof is completed.

Corollary 6. Let $(X, \leq)$ be a partially ordered set and suppose that there exists a metric $d$ on $X$ such that $(X, d)$ is a complete metric space. Let $T: X \rightarrow X$ be a monotone non-decreasing mapping. Suppose that $T$ satisfies for all $x, y \in X$, for which $x \leq y$,

$$
d(T x, T y) \leq \frac{1}{2}[d(x, T y)+d(y, T x)]-\varphi(d(x, T y), d(y, T x)),
$$

where $\varphi:[0,+\infty) \times[0,+\infty) \rightarrow[0,+\infty)$ is a continuous function with $\varphi(x, y)=0$ if and only if $x=y=0$.

Also, suppose either

(i) If $\left\{x_{n}\right\} \subset X$ is a non-decreasing sequence with $x_{n} \rightarrow z$, then $x_{n} \leq z$ for every $n$, or

(ii) $T$ is continuous.

If there exists $x_{0} \in X$ with $x_{0} \leq T x_{0}$, then $T$ has a fixed point.

Proof. It follows by taking $\psi(t)=t$ in Corollary 5 .

Remark 2. Corollary 6 corresponds to Theorem 2.1 and Theorem 2.2 of Harjani et al. [8].

Corollary 7. Let $(X, \leq)$ be a partially ordered set and suppose that there exists a metric $d$ on $X$ such that $(X, d)$ is a complete metric space. Let $T: X \rightarrow X$ be a monotone non-decreasing mapping. Suppose that $T$ satisfies for all $x, y \in X$, for which $x \leq y$,

$$
d(T x, T y) \leq k[d(x, T y)+d(y, T x)]
$$

where $0<k<\frac{1}{2}$.

Also, suppose either

(i) If $\left\{x_{n}\right\} \subset X$ is a non-decreasing sequence with $x_{n} \rightarrow z$, then $x_{n} \leq z$ for every $n$, or

(ii) $T$ is continuous.

If there exists $x_{0} \in X$ with $x_{0} \leq T x_{0}$, then $T$ has a fixed point.

Proof. It follows by taking $\varphi(t)=\left(\frac{1}{2}-k\right) t$ in Corollary 6 . 


\section{REFERENCES}

[1] Y. I. Alber and S. Guerre-Delabriere, "Principle of weakly contractive maps in Hilbert spaces," in New results in operator theory and its applications: the Israel M. Glazman memorial volume, ser. Oper. Theory, Adv. Appl., I. Gohberg, Ed. Basel: Birkhäuser, 1997, vol. 98, pp. 7-22.

[2] S. Banach, "Sur les opérations dans les ensembles abstraits et leur application aux équations intégrales," Fundamenta math., vol. 3, pp. 133-181, 1922.

[3] D. W. Boyd and J. S. W. Wong, "On nonlinear contractions," Proc. Am. Math. Soc., vol. 20, pp. 458-464, 1969.

[4] S. Chandok, "Some common fixed point theorems for generalized $f$-weakly contractive mappings," J. Appl. Math. Inform., vol. 29, no. 1-2, pp. 257-265, 2011.

[5] S. K. Chatterjee, "Fixed point theorem," C. R. Acad. Bulgare Sci., vol. 25, pp. 727-730, 1972.

[6] B. S. Choudhury, "Unique fixed point theorem for weakly $C$-contractive mappings," Kathmandu University J. Sci. Engg. Tech., vol. 5, pp. 6-13, 2009.

[7] L. Ćirić, N. Cakić, M. Rajović, and J. S. Ume, "Monotone generalized nonlinear contractions in partially ordered metric spaces," Fixed Point Theory Appl., vol. 2008, p. 11, 2008.

[8] J. Harjani, B. López, and K. Sadarangani, "Fixed point theorems for weakly $c$-contractive mappings in ordered metric spaces," Comput. Math. Appl., vol. 61, no. 4, pp. 790-796, 2011.

[9] R. Kannan, "Some results on fixed points. ii." Am. Math. Mon., vol. 76, pp. 405-408, 1969.

[10] R. Kannan, "Some results on fixed points," Bull. Calcutta Math. Soc., vol. 60, pp. 71-76, 1986.

[11] M. S. Khan, M. Swaleh, and S. Sessa, "Fixed point theorems by altering distances between the points," Bull. Aust. Math. Soc., vol. 30, pp. 1-9, 1984.

[12] H. K. Nashine and B. Samet, "Fixed point results for mappings satisfying $(\psi, \varphi)$-weakly contractive condition in partially ordered metric spaces," Nonlinear Anal., Theory Methods Appl., Ser. A, Theory Methods, vol. 74, no. 6, pp. 2201-2209, 2011.

[13] J. J. Nieto, R. L. Pouso, and R. Rodríguez-López, "Fixed point theorems in ordered abstract spaces," Proc. Am. Math. Soc., vol. 135, no. 8, pp. 2505-2517, 2007.

[14] J. J. Nieto and R. Rodríguez-López, "Contractive mapping theorems in partially ordered sets and applications to ordinary differential equations," Order, vol. 22, no. 3, pp. 223-239, 2005.

[15] O. Popescu, "Fixed points for $(\psi, \varphi)$-weak contractions," Appl. Math. Lett., vol. 24, no. 1, pp. 1-4, 2011.

[16] A. C. M. Ran and M. C. B. Reurings, "A fixed point theorem in partially ordered sets and some applications to matrix equations," Proc. Am. Math. Soc., vol. 132, no. 5, pp. 1435-1443, 2004.

[17] B. E. Rhoades, "Some theorems on weakly contractive maps," Nonlinear Anal., Theory Methods Appl., vol. 47, no. 4, pp. 2683-2693, 2001.

[18] W. Shatanawi, "Fixed point theorems for nonlinear weakly-contractive mappings in metric spaces," Math. Comput. Modelling, vol. 54, no. 11-12, pp. 2816-2826, 2011.

[19] Q. Zhang and Y. Song, "Fixed point theory for generalized $\varphi$-weak contractions," Appl. Math. Lett., vol. 22, no. 1, pp. 75-78, 2009.

\section{Author's address}

\section{H. Aydi}

Université de Sousse, Institut Supérieur d'Informatique et des Technologies de Communication de Hammam Sousse, Route GP1-4011, H. Sousse, Tunisia

E-mail address: hassen.aydi@isima.rnu.tn 